\title{
Infinite-Series Representations Associated With the Bivariate Rician Distribution and Their Applications
}

\author{
Dimitris A. Zogas, Member, IEEE, and George K. Karagiannidis, Senior Member, IEEE
}

\begin{abstract}
Analytical expressions for the evaluation of the bivariate Rician cumulative distribution function (CDF), the covariance, and the characteristic function (CHF) are not known, despite their usefulness in wireless communications systems analysis. In this letter, motivated by the ability of the Rician model to describe fading in wireless communications, we derive infinite-series representations for the probability density function, the CDF, the covariance, and the CHF of two correlated Rician random variables. It is shown that the presented infinite-series expressions converge rapidly, and can be efficiently used to study several performance criteria for dual-diversity receivers operating over correlated Rician fading channels.
\end{abstract}

Index Terms-Communications channels, correlated fading, digital communications, fading channels, Rician fading.

\section{INTRODUCTION}

$\mathbf{S}$ EVERAL statistical models are used in communications systems analysis to describe fading in wireless environments. The most frequently used distributions are Rayleigh, Nakagami- $m$, Rice, and Weibull. Moreover, several problems in wireless communications theory involve bivariate and, in the general case, multivariate distributions. Examples of such problems can be found in the performance analysis of correlative fading applications with space or frequency diversity, in multichannel reception, or in the transition probabilities in a first (or higher) order Markov chain that models the fading process [1].

Looking at the open up-to-date technical literature, several approaches are presented concerning the multivariate Rayleigh and Nakagami- $m$ distributions. More specifically, Nakagami presented the bivariate Rayleigh and Nakagami- $m$ probability density function (PDF) [2]. Tan and Beaulieu [1] proposed an infinite-series representation for the bivariate Nakagami- $m$ cumulative distribution function (CDF), and Simon and Alouini [3] formulated the bivariate Rayleigh CDF with a single integral with finite limits. Concerning the multivariate Rayleigh and Nakagami- $m$ distributions, Mallik [4] presented analytical results for the multivariate Rayleigh distribution, while Karagiannidis et al. [5], [6] derived expressions for the multivariate Nakagami- $m$ PDF and CDF. Moreover, the covariance of correlated Nakagami- $m$ random variables (RVs) is presented in [7]. Most of these works have been used to study, for example, the effects of correlated fading on the performance

Paper approved by M.-S. Alouini, the Editor for Modulation and Diversity Systems of the IEEE Communications Society. Manuscript received October 27, 2004; revised April 21, 2005 and June 14, 2005.

The authors are with the Department of Electrical and Computer Engineering, Division of Telecommunications, Aristotle University of Thessaloniki, Thessaloniki 54 124, Greece (e-mail: geokarag@auth.gr; geokarag@ieee.org; zogas@ space.noa.gr).

Digital Object Identifier 10.1109/TCOMM.2005.858659 of wireless communications systems with diversity at the receiver [8]-[12]. However, despite the usefulness of the Rice model, only the bivariate Rician PDF was given in the past as [8, eq. (15)]

$$
\begin{aligned}
f_{x_{1}, x_{2}}\left(x_{1}, x_{2}\right)= & \frac{(1+K)^{2} x_{1} x_{2}}{2 \pi \beta^{2}\left(1-u^{2}\right)} \exp \left[\frac{-2 K}{1+u}\right] \\
& \times \exp \left[-\frac{(1+K)\left(x_{1}^{2}+x_{2}^{2}\right)}{2\left(1-u^{2}\right) \beta}\right] \\
& \times \int_{0}^{2 \pi} \exp \left[\frac{u(1+K) x_{1} x_{2} \cos \theta}{\left(1-u^{2}\right) \beta}\right] \\
& \times I_{0}\left(\sqrt{\frac{2 K\left(x_{1}^{2}+x_{2}^{2}+2 x_{1} x_{2} \cos \theta\right)}{\beta(1+K)^{-1}(1+u)^{2}}}\right) d \theta
\end{aligned}
$$

where $x_{1}=|W|$ and $x_{2}=|H|$ are correlated Rician RVs, with $W, H$ as nonzero-mean correlated complex Gaussian RVs, $u$ is the correlation coefficient, which is assumed to be real, and is defined as

$$
u=\frac{\left\langle\left(W^{*}-\left\langle W^{*}\right\rangle\right)(H-\langle H\rangle)\right\rangle}{\sqrt{\left\langle|W-\langle W\rangle|^{2}\right\rangle} \sqrt{\left\langle|H-\langle H\rangle|^{2}\right\rangle}}
$$

where

$\langle\cdot\rangle \quad$ expectation;

$\beta \quad$ average power of $x_{1}, x_{2}$ defined as $\beta=\overline{x_{1}^{2}} / 2=\overline{x_{2}^{2}} / 2$;

$K \quad$ Rice factor defined as the ratio of the signal power in the dominant component over the scattered power;

$I_{0}(\cdot)$ modified Bessel function of the first kind and zeroth order.

The complicated form of (1) was the reason for the use of numerical integration in [8] and [13] in order to study the error performance of dual switched diversity receivers over correlated Rician flat-fading channels, and of orthogonal frequency-division multiplexing (OFDM) systems over frequency-nonselective fast Rician channels, respectively.

In this letter, motivated by the importance of the Rician model to describe fading in wireless communications systems, we present a novel infinite-series representation for the bivariate Rician PDF. This formula is used to derive similar expressions with rapid convergence for the $\mathrm{CDF}$, the covariance, and the characteristic function (CHF) of two correlated identically distributed (i.d.) Rician RVs. The proposed expressions can be used to study several performance criteria, such as the outage probability and the average error rate for different modulation schemes, of dual-diversity receivers employing selection combining (SC), equal-gain combining (EGC), and 
switched-and-stay combining (SSC). However, due to the limited size of this letter, we present, as an indicative example of the mathematical analysis, the evaluation of the outage probability of a dual SC receiver operating over correlated Rician fading channels.

\section{INFINITE-SERIES REPRESENTATIONS}

\section{A. Probability Density Function}

Using the infinite-series representation of $I_{0}(\cdot)$ [14, eq. $(8.445)]$

$$
I_{0}(z)=\sum_{k=0}^{\infty} \frac{z^{2 k}}{2^{2 k} k ! \Gamma(k+1)}
$$

and changing the order of summation and integration, (1) can be written as

$$
\begin{aligned}
f_{x_{1}, x_{2}}\left(x_{1}, x_{2}\right)= & \sum_{i=0}^{\infty} \frac{(1+K)^{2}}{2 \pi \beta^{2}\left(1-u^{2}\right)}\left[\frac{K(1+K)}{\beta(1+u)^{2}}\right]^{i} \\
& \times \frac{x_{1} x_{2}}{2^{i} i ! \Gamma(i+1)} \\
& \times \exp \left[\frac{-2 K}{1+u}-\frac{(1+K)\left(x_{1}^{2}+x_{2}^{2}\right)}{2\left(1-u^{2}\right) \beta}\right] \\
& \times \int_{0}^{2 \pi} \exp \left[\frac{u(1+K) x_{1} x_{2} \cos \theta}{\left(1-u^{2}\right) \beta}\right] \\
& \times\left(x_{1}^{2}+x_{2}^{2}+2 x_{1} x_{2} \cos \theta\right)^{i} d \theta .
\end{aligned}
$$

Expanding the term $\left(x_{1}^{2}+x_{2}^{2}+2 x_{1} x_{2} \cos \theta\right)^{i}$ using the multinomial identity results in

$$
\begin{aligned}
f_{x_{1}, x_{2}}\left(x_{1}, x_{2}\right)= & \sum_{\substack{i=0 \\
v_{1}+v_{2}+v_{3}=i}}^{\infty} \frac{(1+K)^{2}}{2 \pi \beta^{2}\left(1-u^{2}\right)}\left[\frac{K(1+K)}{\beta(1+u)^{2}}\right]^{i} \\
& \times \frac{2^{v_{3}} x_{1}^{2 v_{1}+v_{3}+1} x_{2}^{2 v_{2}+v_{3}+1}}{2^{i} v_{1} ! v_{2} ! v_{3} ! \Gamma(i+1)} \\
& \times \exp \left[\frac{-2 K}{1+u}-\frac{(1+K)\left(x_{1}^{2}+x_{2}^{2}\right)}{2\left(1-u^{2}\right) \beta}\right] \\
& \times \int_{0}^{2 \pi} \exp \left[\frac{u(1+K) x_{1} x_{2} \cos \theta}{\left(1-u^{2}\right) \beta}\right] \\
& \times(\cos \theta)^{v_{3}} d \theta
\end{aligned}
$$

with $v_{1}, v_{2}, v_{3}$ being nonnegative integers. Letting $x=\cos \theta$ and after manipulations, the integral in (5) can be solved with the use of [14, eq. (3.389/1)] resulting in

$$
\begin{aligned}
& f_{x_{1}, x_{2}}\left(x_{1}, x_{2}\right) \\
& =\sum_{\substack{i=0 \\
v_{1}+v_{2}+v_{3}=i}}^{\infty} \mathcal{A}\left[\mathcal{D}_{1} x_{1}^{2 v_{1}+v_{3}+1} x_{2}^{2 v_{2}+v_{3}+1} \exp \left[-\mathcal{E}\left(x_{1}^{2}+x_{2}^{2}\right)\right]\right. \\
& \quad \times_{1} F_{2}\left(\frac{1+v_{3}}{2} ; \frac{1}{2}, \frac{2+v_{3}}{2} ; \mathcal{F} x_{1}^{2} x_{2}^{2}\right) \\
& \quad+\mathcal{D}_{2} x_{1}^{2 v_{1}+v_{3}+1} x_{2}^{2 v_{2}+v_{3}+1} \exp \left[-\mathcal{E}\left(x_{1}^{2}+x_{2}^{2}\right)\right] \\
& \left.\quad \times_{1} F_{2}\left(\frac{2+v_{3}}{2} ; \frac{3}{2}, \frac{3+v_{3}}{2} ; \mathcal{F} x_{1}^{2} x_{2}^{2}\right)\right]
\end{aligned}
$$

where

$$
\begin{aligned}
\mathcal{A} & =\frac{2^{v_{3}-i-2}(1+K)^{2+i} K^{i}(1+u)^{-2 i}}{\beta^{2+i}\left(1-u^{2}\right) v_{1} ! v_{2} ! v_{3} ! \Gamma(i+1)} \exp \left(-\frac{2 K}{1+u}\right) \\
\mathcal{D}_{1} & =2\left(1+(-1)^{v_{3}}\right) \frac{\Gamma\left(\frac{1+v_{3}}{2}\right)}{\sqrt{\pi} \Gamma\left(\frac{2+v_{3}}{2}\right)} \\
\mathcal{D}_{2} & =\frac{2\left(-1+(-1)^{v_{3}}\right) u(1+K) \Gamma\left(\frac{2+v_{3}}{2}\right)}{\sqrt{\pi} \beta\left(u^{2}-1\right) \Gamma\left(\frac{3+v_{3}}{2}\right)} \\
\mathcal{E} & =\frac{1+K}{2 \beta\left(1-u^{2}\right)} \\
\mathcal{F} & =\frac{(1+K)^{2} u^{2}}{4 \beta^{2}(u-1)^{2}(1+u)^{2}} .
\end{aligned}
$$

Note that after the truncation of the infinite series in (6) at the value $i=I-1$, the remainder $R_{I}$ converges to zero as $I$ increases (for the proof, see the Appendix).

\section{B. Cumulative Distribution Function}

The bivariate Rician CDF is by definition

$$
F_{x_{1}, x_{2}}\left(x_{1}, x_{2}\right)=\int_{0}^{x_{1}} \int_{0}^{x_{2}} f_{x_{1}, x_{2}}\left(y_{1}, y_{2}\right) d y_{1} d y_{2} .
$$

In order to solve the integrals in (7), we substitute the hypergeometric functions of (6) with their infinite-series representation [14, eq. (9.14/1)], i.e.,

$$
{ }_{1} F_{2}\left(p ; q_{1}, q_{2} ; x\right)=\sum_{h=0}^{\infty} \frac{(p)_{h} x^{h}}{\left(q_{1}\right)_{h}\left(q_{2}\right)_{h} h !}
$$

with $(\cdot)_{n}$ denoting the Pochhammer symbols [15, eq. (6.1.22)]. This leads to a new formula for the bivariate PDF, shown in (9) at the bottom of the page, where

$$
\mathcal{G}_{1}=\frac{\left(\frac{1+v_{3}}{2}\right)_{h}}{\left(\frac{1}{2}\right)_{h}\left(\frac{2+v_{3}}{2}\right)_{h} h !}, \quad \mathcal{G}_{2}=\frac{\left(\frac{2+v_{3}}{2}\right)_{h}}{\left(\frac{3}{2}\right)_{h}\left(\frac{3+v_{3}}{2}\right)_{h} h !} .
$$

$$
\begin{aligned}
f_{x_{1}, x_{2}}\left(x_{1}, x_{2}\right)= & \sum_{\substack{i, h=0 \\
v_{1}+v_{2}+v_{3}=i}}^{\infty} \mathcal{A} \mathcal{F}^{h}\left[\mathcal{D}_{1} \mathcal{G}_{1} x_{1}^{2 v_{1}+v_{3}+2 h+1} x_{2}^{2 v_{2}+v_{3}+2 h+1} \exp \left[-\mathcal{E}\left(x_{1}^{2}+x_{2}^{2}\right)\right]\right. \\
& \left.+\mathcal{D}_{2} \mathcal{G}_{2} x_{1}^{2 v_{1}+v_{3}+2 h+2} x_{2}^{2 v_{2}+v_{3}+2 h+2} \exp \left[-\mathcal{E}\left(x_{1}^{2}+x_{2}^{2}\right)\right]\right]
\end{aligned}
$$


Substituting (9) into (7) and interchanging the order of summation and integration, integrals of the form

$$
\int_{0}^{x} y^{n} \exp \left(-z y^{2}\right) d y
$$

are produced. This integral can be solved by making the transformation $t=z y^{2}$ and using the definition of the "lower" incomplete Gamma function [15, eq. (6.5.2)], i.e.,

$$
\gamma(p, x)=\int_{0}^{x} y^{p-1} \exp (-y) d y
$$

resulting finally in (12), shown at the bottom of the page.

\section{Covariance}

The covariance of $x_{1}, x_{2}$ is defined as

$$
\operatorname{cov}\left\langle x_{1}^{n_{1}} x_{2}^{n_{2}}\right\rangle=\int_{0}^{\infty} \int_{0}^{\infty} x_{1}^{n_{1}} x_{2}^{n_{2}} f_{x_{1}, x_{2}}\left(x_{1}, x_{2}\right) d x_{1} d x_{2} .
$$

Similar to the derivation of the bivariate CDF, substituting (9) into (13) and interchanging the order of summation and integration, integrals of the form

$$
\int_{0}^{\infty} y^{n} \exp \left(-z y^{2}\right) d y
$$

are produced. The above integrals can be solved using [14, eq. (3.461/3)], and after manipulations, the covariance of $x_{1}, x_{2}$ is finally given by (15), shown at the bottom of the page.

\section{Characteristic Function}

The CHF of $x_{1}, x_{2}$ is, by definition

$$
\begin{aligned}
\Phi_{x_{1}, x_{2}}\left(s_{1}, s_{2}\right)=\int_{0}^{\infty} & \int_{0}^{\infty} f_{x_{1}, x_{2}}\left(x_{1}, x_{2}\right) \\
& \times \exp \left(j s_{1} x_{1}+j s_{2} x_{2}\right) d x_{1} d x_{2}
\end{aligned}
$$

with $j=\sqrt{-1}$. Substituting (9) into (16), integrals of the form

$$
\int_{0}^{\infty} y^{n} \exp \left(-z_{1} y^{2}-z_{2} y\right) d y
$$

are produced, which can be solved using [14, eq. (3.462/2)], and $\Phi_{x_{1}, x_{2}}\left(s_{1}, s_{2}\right)$ can be finally written as

$$
\begin{aligned}
\Phi_{x_{1}, x_{2}}\left(s_{1}, s_{2}\right)= & \sum_{\substack{i, h=0 \\
v_{1}+v_{2}+v_{3}=i}}^{\infty} \frac{\mathcal{A} \mathcal{F}^{h}}{(2 \mathcal{E})^{i+2 h+2}} \\
& \times \exp \left(-\frac{s_{1}^{2}+s_{2}^{2}}{8 \mathcal{E}}\right) \\
\times & {\left[\mathcal{D}_{1} \mathcal{G}_{1} g\left(z_{1}, t_{1}\right) g\left(z_{2}, t_{2}\right)+\frac{\mathcal{D}_{2} \mathcal{G}_{2}}{2 \mathcal{E}}\right.} \\
& \left.\quad \times g\left(z_{1}+1, t_{1}\right) g\left(z_{2}+1, t_{2}\right)\right]
\end{aligned}
$$

where

$$
\begin{aligned}
g(z, t) & =\Gamma(z) D_{-z}(t) \\
z_{1} & =2 v_{1}+v_{3}+2 h+2, \quad t_{1}=-\frac{j s_{1}}{\sqrt{2 \mathcal{E}}} \\
z_{2} & =2 v_{2}+v_{3}+2 h+2, \quad t_{2}=-\frac{j s_{2}}{\sqrt{2 \mathcal{E}}}
\end{aligned}
$$

with $D_{-v}(z)$ being the parabolic cylinder function of order $v$ and argument $z$ [15, eq. (9.240)].

\section{APPLICATIONS IN DUAL-DIVERSITY RECEIVERS}

The proposed infinite-series representations (12), (15), and (18) can be efficiently used to study important performance criteria, such as the outage probability and the average error rate for several modulation schemes, of dual-diversity receivers operating over correlated Rician fading channels.

1) Selection Combining: The formula (12) for the joint Rician CDF can be directly applied for the study of the outage performance of dual SC receivers. Let $\gamma_{i}=x_{i}^{2} E_{s} / 2 N_{0}$ and $\bar{\gamma}_{i}=\beta E_{s} / N_{0}$, with $E_{s} / N_{0}$ being the symbol energy-to-Gaussian noise spectral density ratio, denote the instantaneous and mean signal-to-noise ratio (SNR) at the

$$
\begin{aligned}
F_{x_{1}, x_{2}}\left(x_{1}, x_{2}\right)= & \sum_{\substack{i, h=0 \\
v_{1}+v_{2}+v_{3}=i}}^{\infty} \frac{\mathcal{A} \mathcal{F}^{h}}{4 \mathcal{E}^{i+2 h+2}}\left[\mathcal{D}_{1} \mathcal{G}_{1} \gamma\left(v_{1}+\frac{v_{3}}{2}+h+1, \mathcal{E} x_{1}^{2}\right) \gamma\left(v_{2}+\frac{v_{3}}{2}+h+1, \mathcal{E} x_{2}^{2}\right)\right. \\
& \left.+\frac{\mathcal{D}_{2} \mathcal{G}_{2}}{\mathcal{E}} \gamma\left(v_{1}+\frac{v_{3}}{2}+h+\frac{3}{2}, \mathcal{E} x_{1}^{2}\right) \gamma\left(v_{2}+\frac{v_{3}}{2}+h+\frac{3}{2}, \mathcal{E} x_{2}^{2}\right)\right]
\end{aligned}
$$

$$
\begin{aligned}
\operatorname{cov}\left\langle x_{1}^{n_{1}} x_{2}^{n_{2}}\right\rangle= & \sum_{\substack{i, h=0 \\
v_{1}+v_{2}+v_{3}=i}}^{\infty} \frac{\mathcal{A F}^{h}}{4 \mathcal{E}^{i+2 h+2+\left(n_{1}+n_{2} / 2\right)}}\left[\mathcal{D}_{1} \mathcal{G}_{1} \Gamma\left(v_{1}+\frac{v_{3}}{2}+h+\frac{n_{1}}{2}+1\right) \Gamma\left(v_{2}+\frac{v_{3}}{2}+h+\frac{n_{2}}{2}+1\right)\right. \\
& \left.+\frac{\mathcal{D}_{2} \mathcal{G}_{2}}{\mathcal{E}} \Gamma\left(v_{1}+\frac{v_{3}}{2}+h+\frac{n_{1}}{2}+\frac{3}{2}\right) \Gamma\left(v_{2}+\frac{v_{3}}{2}+h+\frac{n_{2}}{2}+\frac{3}{2}\right)\right]
\end{aligned}
$$


TABLE I

NUMBER OF TERMS OF (12) REQUIRED FOR SIX-SIGNIFICANT-FIGURE ACCURACY

\begin{tabular}{|c|c|c|c|c|c|c|}
\hline \multirow[b]{2}{*}{$u$} & \multicolumn{3}{|c|}{$K=0 \mathrm{~dB}$} & \multicolumn{3}{|c|}{$K=7 \mathrm{~dB}$} \\
\hline & $\begin{array}{l}x_{1}=1 \\
\mathrm{x}_{2}=1\end{array}$ & $\begin{array}{l}x_{1}=1 \\
x_{1}=5\end{array}$ & $\begin{array}{l}\mathrm{x}_{2}=5 \\
\mathrm{x}_{2}=5\end{array}$ & $\begin{array}{l}x_{1}=1 \\
\mathrm{x}_{2}=1\end{array}$ & $\begin{array}{l}x_{1}=1 \\
x_{1}=5\end{array}$ & $\begin{array}{l}\mathrm{x}_{2}=5 \\
\mathrm{x}_{2}=5\end{array}$ \\
\hline 0.1 & 6 & 8 & 11 & 14 & 20 & 26 \\
\hline 0.3 & 5 & 7 & 11 & 12 & 18 & 26 \\
\hline 0.6 & 5 & 7 & 17 & 11 & 14 & 29 \\
\hline 0.9 & 10 & 15 & 63 & 23 & 32 & 124 \\
\hline
\end{tabular}

$k$-branch $(k=1,2)$, respectively, of a dual SC receiver operating over correlated Rician fading channels. The outage probability of the SC output SNR, $P_{\text {out }}$, can be evaluated as [9]

$$
\begin{aligned}
P_{\text {out }} & =F_{\gamma_{\text {out }}}\left(\gamma_{t h}\right)=F_{\gamma_{1}, \gamma_{2}}\left(\gamma_{t h}, \gamma_{t h}\right) \\
& =F_{x_{1}, x_{2}}\left(\sqrt{2 \beta \frac{\gamma_{t h}}{\bar{\gamma}}}, \sqrt{2 \beta \frac{\gamma_{t h}}{\bar{\gamma}}}\right) .
\end{aligned}
$$

2) Equal-Gain Combining: The CHF of two correlated Rician RVs is needed to study the binary phase-shift keying (BPSK) error performance of dual predetection ECG receivers, based on the Gil-Pelaez inversion theorem [11]. Similarly, the joint CHF can be used to find expressions for the CHF of the sum of correlated RVs to study the EGC error performance for several modulation schemes [10], [16]. The covariance of two correlated Rician RVs can be used to evaluate significant performance criteria of the EGC output SNR, such as the average output SNR, the amount of fading (AoF), the higher order moments, and the outage and error performance for a broad range of modulation schemes, using the moments-based approach [7].

3) Switched-and-Stay Combining: The infinite series for the joint CDF can be used to study the error performance of switched-and-stay diversity receivers, applying the well-known Beaulieu series approach [8].

\section{NUMERICAL RESULTS}

In order to check the accuracy of the proposed mathematical analysis, the results obtained by the infinite series in (12) are compared with the corresponding ones from (7), where the involved integrals are evaluated numerically. Table I gives the results for six-significant-figure accuracy, assuming, without loss of generality, that $h=i$, with $\beta$ normalized to one and for a range of values for $x_{1}, x_{2}, K, u$. We observe that an increase of $K$ or $x_{1}, x_{2}$ leads to an increase of the required terms that need to be summed to obtain a target accuracy. Furthermore, comparing with the convergence of the infinite-series representation for the bivariate or multivariate Nakagami- $m$ CDF [1], [5], where an increase of the correlation leads to an increase of the required terms needing to be summed; for the bivariate Rician case, only for high correlation values the required terms increase. Moreover, even for the highest correlation situation $(u \rightarrow 1)$, the required terms are much less than the corresponding ones for the Nakagami- $m$ case.

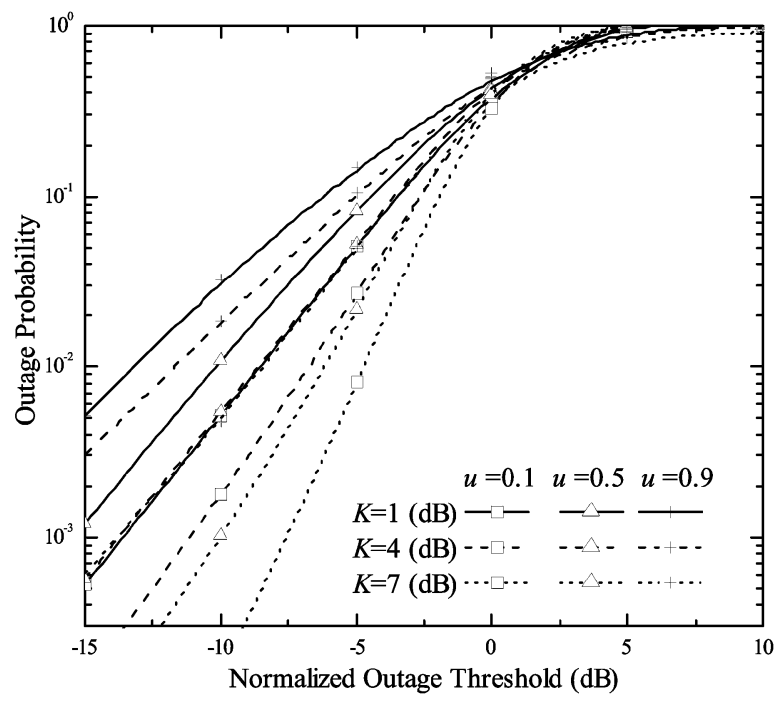

Fig. 1. Outage probability $P_{\text {out }}$ versus the normalized outage threshold $\gamma_{t h} / \bar{\gamma}$ for a dual SC receiver and for several values of $K$ and $u$.

Using (19), the outage performance of a dual SC receiver operating in correlated Rician fading channels is plotted in Fig. 1. The obtained results show clearly that the outage performance degrades with an increase of the fading correlation and/or the fading severity.

\section{CONCLUSIONS}

We derived infinite-series representations for the PDF, the $\mathrm{CDF}$, the covariance, and the CHF of two correlated i.d. Rician $\mathrm{RV}$ s. It is shown that the presented infinite-series expressions converge rapidly, and thus, they can efficiently be used to analytically study several performance criteria of dual-diversity receivers when correlated Rician fading is assumed. We investigated, as an indicative example, the outage probability of a dual $\mathrm{SC}$ receiver where the numerical results clearly depict the effect of the fading correlation and the fading severity on the receiver's performance.

\section{APPENDIX \\ CONVERGENCE OF THE INFINITE-SERIES REPRESENTATIONS}

After the truncation of the infinite series in (6) at the value $i=I-1$, the remainder $R_{I}$ converges to zero as $I$ increases, since the term $\mathcal{A}$ converges to zero for any combination of $v_{1}$, $v_{2}, v_{3}$ which satisfies $v_{1}+v_{2}+v_{3} \rightarrow \infty$. This can be proved as follows.

The term $\mathcal{A}$ can be upper bounded by

$$
\begin{aligned}
\mathcal{A} \leq \mathcal{A}^{\prime}= & \frac{2^{-v_{1}-v_{2}-2}(1+K)^{2+v_{1}+v_{2}+v_{3}} K^{v_{1}+v_{2}+v_{3}}}{\beta^{2+v_{1}+v_{2}+v_{3}}\left(1-u^{2}\right) v_{1} ! v_{2} ! v_{3} ! \Gamma\left(v_{3}+1\right)} \\
& \times(1+u)^{-2 v_{1}-2 v_{2}-2 v_{3}} \exp \left(-\frac{2 K}{1+u}\right)
\end{aligned}
$$

and $\mathcal{A}^{\prime}$ can be written as

$$
\begin{array}{r}
\mathcal{A}^{\prime}=\frac{2^{-v_{1}-v_{2}-2}(1+K)^{2+v_{1}+v_{2}} K^{v_{1}+v_{2}}(1+u)^{-2 v_{1}-2 v_{2}}}{\beta^{2+v_{1}+v_{2}}\left(1-u^{2}\right) v_{1} ! v_{2} !} \\
\times \exp \left(-\frac{2 K}{1+u}\right)\left(\frac{K(K+1)}{b(1+u)^{2}}\right)^{v_{3}} \frac{1}{v_{3} !^{2}} .
\end{array}
$$


If $v_{1}=n, v_{2}=n, v_{3} \rightarrow \infty$ (similar if $v_{1} \rightarrow \infty, v_{2}=n$, $v_{3}=n$, or $\left.v_{1}=n, v_{2} \rightarrow \infty, v_{3}=n\right)$, then

$$
\lim _{v_{3} \rightarrow \infty} \mathcal{A}^{\prime}=\lim _{v_{3} \rightarrow \infty}\left(\frac{K(K+1)}{b(1+u)^{2}}\right)^{v_{3}} \frac{1}{v_{3} !^{2}} .
$$

The limit in (22) has the form

$$
\lim _{t \rightarrow \infty} \frac{Z^{t}}{t !}=0
$$

with $Z$ being real and $t$ integer. Therefore, from (20)-(23), results

$$
\lim _{v_{3} \rightarrow \infty} \mathcal{A}^{\prime}=0 \Rightarrow \lim _{v_{3} \rightarrow \infty} \mathcal{A}=0 \Rightarrow \lim _{v_{3} \rightarrow \infty} R_{I}=0
$$

The same analysis can be also applied for the infinite-series representations of the $\mathrm{CDF}$, covariance, and $\mathrm{CHF}$ to prove their convergence.

\section{ACKNOWLEDGMENT}

The authors would like to thank Mr. A. K. Zogas for his helpful comments on the convergence of the infinite-series representations.

\section{REFERENCES}

[1] C. C. Tan and N. C. Beaulieu, "Infinite-series representations of the bivariate Rayleigh and Nakagami- $m$ distribution," IEEE Trans. Commun., vol. 45, no. 10, pp. 1159-1161, Oct. 1997.

[2] M. Nakagami, "The $m$-distribution-A general formula of intensity distribution of rapid fading," in Statistical Methods in Radio Wave Propagation, W. C. Hofman, Ed. New York: Pergamon, 1960.
[3] M. K. Simon and M.-S. Alouini, "A simple single integral representation of the bivariate Rayleigh distribution," IEEE Commun. Lett., vol. 2, no. 5, pp. 128-130, May 1998.

[4] R. K. Mallik, "On the multivariate Rayleigh and exponential distributions," IEEE Trans. Inf. Theory, vol. 49, no. 6, pp. 1499-1515, Jun. 2003

[5] G. K. Karagiannidis, D. A. Zogas, and S. A. Kotsopoulos, "On the multivariate Nakagami- $m$ distribution with exponential correlation," IEEE Trans. Commun., vol. 51, no. 8, pp. 1240-1244, Aug. 2003.

[6] _ - "An efficient approach to multivariate Nakagami- $m$ distribution using Green's matrix approximation," IEEE Trans. Wireless Commun., vol. 2, no. 9, pp. 883-889, Sep. 2003.

[7] G. K. Karagiannidis, "Moments-based approach to the performance analysis of equal gain diversity in Nakagami- $m$ fading," IEEE Trans. Commun., vol. 52, no. 5, pp. 685-690, May 2004.

[8] A. A. Abu-Dayya and N. C. Beaulieu, "Switched diversity on microcellular Ricean channels," IEEE Trans. Veh. Technol., vol. 43, no. 6, pp. 970-976, Nov. 1994.

[9] G. K. Karagiannidis, D. A. Zogas, and S. A. Kotsopoulos, "Performance analysis of triple selection diversity over exponentially correlated Nakagami- $m$ fading channels," IEEE Trans. Commun., vol. 51, no. 8, pp. 1245-1248, Aug. 2003.

[10] - "BER performance of dual predetection EGC in correlative Nakagami- $m$ fading," IEEE Trans. Commun., vol. 52, no. 1, pp. 50-53, Jan. 2004.

[11] R. K. Mallik, M. Z. Win, and J. H. Winters, "Performance of dual-diversity predetection EGC in correlated Rayleigh fading with unequal branch SNRs," IEEE Trans. Commun., vol. 50, no. 7, pp. 1041-1044, Jul. 2002.

[12] M. K. Simon and M.-S. Alouini, "A unified performance analysis of digital communication with dual selective combining diversity over correlated Rayleigh and Nakagami- $m$ fading channels," IEEE Trans. Commun., vol. 47, no. 1, pp. 33-43, Jan. 1999.

[13] L. Wan and V. K. Dubey, "BER performance of OFDM system over frequency nonselective fast Rician fading channels," IEEE Commun. Lett., vol. 5, no. 1, pp. 19-21, Jan. 2001.

[14] I. S. Gradshteyn and I. M. Ryzhik, Table of Integrals, Series, and Products, 6th ed. New York: Academic, 1994

[15] M. Abramowitz and I. A. Stegun, Handbook of Mathematical Functions With Formulas, Graphs, and Mathematical Tables, 9th ed. New York: Dover, 1972.

[16] A. Annamalai, C. Tellambura, and V. K. Bhargava, "Equal-gain diversity receiver performance in wireless channels," IEEE Trans. Commun., vol. 48, no. 10 , pp. 1732-1745, Oct. 2000. 